Rozmahel, J.L. Cole, et al. 1989. Identification of the cystic fibrosis gene: chromosome walking and jumping. Science 245:1059-1065.

5.Tsui, L.C. 1992. Mutations and sequence variations detected in the cystic fibrosis transmembrane conductance regulator (CFTR) gene: a report from the Cystic Fibrosis Genetic Analysis Consortium. Hum. Mutat. 1:197-203.

6.Ballabio, A., R.A. Gibbs, and C.T. Caskey. 1990. PCR test for cystic fibrosis deletion. Nature 343:220.

7.Liu, Q., E.C. Thorland, J.A. Heit, and S.S. Sommer. 1997. Overlapping PCR for bidirectional PCR amplification of specific alleles: a rapid one-tube method for simultaneously differentiating homozygotes and heterozygotes. Genome Res. 7:389-398.

8.Sarkar, G. and S.S. Sommer. 1990. The "megaprimer" method of site-directed mutagenesis. BioTechniques 8:404-407.

9.Sarkar, G. and S.S. Sommer. 1992. Doublestranded DNA segments can efficiently prime the amplification of human genomic DNA. Nucleic Acids Res. 20:4937-4938.

10.Kerem, B.S., J. Zielenski, D. Markiewicz, D. Bozon, E. Gazit, J. Yahav, D. Kennedy, J.R. Riordan, et al. 1990. Identification of mutations in regions corresponding to the two putative nucleotide (ATP)-binding folds of the cystic fibrosis gene. Proc. Natl. Acad. Sci. USA $87: 8447-8451$

Received 15 October 2002; accepted 19 December 2002.

P. Maciel ${ }^{1,2}$, J. Yan ${ }^{1}$, J. Feng ${ }^{1}$, F. Accurso ${ }^{3}$, and S. Sommer ${ }^{1}$ ${ }^{1}$ City of Hope National Medical Center/Beckman Research Institute

Duarte, CA, USA

${ }^{2}$ University of Porto

Porto, Portugal

${ }^{3}$ Children's Hospital

Denver, CO, USA

\section{High-Throughput Inducible Expression of Transgenes at the Hprt Gene in Mouse Embryonic Stem Cells}

BioTechniques 34:462-468 (March 2003)

Overexpression and/or ectopic expression of gene products has led to important discoveries about gene product function; unfortunately, the expression of transgenes has been difficult and unreliable because of our poor understanding of transcription. Pronuclear injection has been the most common method to introduce transgenes into mouse embryos; however, the problems that arise from pronuclear injection are well described (1). First, chromosomal deletions, inversions, and duplications are frequently generated with transgene integration. These alterations could potentially mutate nearby genes, resulting in an unwanted phenotype that complicates analysis of the transgene product (2). Second, variable levels and patterns of transgene expression are observed between founder mice due to concatemerization of the transgene and chromatin position effects (3). Third, mosaic expression of the transgene may occur because of position-effect variegation $(4,5)$, methylation (6), or intrachromosomal recombination within the transgene concatemer (7). Transgenic mice derived from multiple founders must be analyzed to control for these variables, substantially increasing the resources devoted to an experiment. Transgenes can also be introduced directly into cells or mouse embryos by a variety of techniques including retroviral and adenoviralmediated gene transfer and DNA:lipopolyamine-mediated gene transfer. However, these procedures still present many of the same problems as pronuclear injection, in particular, sustained expression of the transgene.

To address these problems, a procedure has been developed termed targeted transgenesis (8). This procedure introduces a single copy of a transgene into a known location and relies on manipulation of embryonic stem (ES) cells in tis- sue culture. Different methods to achieve targeted transgenesis have been developed that include targeting a transgene to the hypoxanthine phosphoribosyltransferase (Hprt) gene (9) and to other loci, as reviewed by Jasin et al. (8). Hprt was chosen because it is a housekeeping gene, expressed in all cells, and functions in the purine salvage pathway. Thus, chromatin effects are likely minimal. In addition, Hprt is on the $\mathrm{X}$ chromosome and therefore is hemizygous in male cells. Disruption of Hprt can be selected in 6-thioguanine (TG) media and expression of Hprt can be selected in hypoxanthine-aminopterin-thymidine (HAT) media. This selection strategy enables high-throughput transgenesis by gene targeting. The fact that all transgenes are positioned at the exact same location in the genome controls for unknown variables of position effect that accompany random integration.

Previously, we have targeted transgenes to Hprt in mouse ES cells; this approach mutated the Hprt gene (9). Our data showed that a reporter was expressed in greater than $85 \%$ of Hprtmutant ES cell clones. In addition, another reporter exhibited the correct temporal and tissue-specific expression pattern of the promoter in mouse embryos. Thus, targeted transgenesis was successful at the Hprt gene.

Here our goal is to establish a highthroughput method for inducible transgene expression in cells and mice with the tetracycline responsive system (10). The tet-responsive activator system is composed of the tet repressor fused to the activation domain of virion protein 16 (VP16) of herpes simplex virus. This transactivator, called $t \mathrm{TA}$, stimulates transcription of a minimal promoter fused to tet operator sequences $($ tet $\mathrm{O})$. The minimal promoter is the minimal immediate early promoter of the cytomegalovirus (mCMV) juxtaposed to the tet $O$ sequences (tet $O$ mCMV). Inducible expression is achieved by the use of a derivative of tetracycline called doxycycline, which binds and inactivates $t \mathrm{TA}$. Thus, $t \mathrm{TA}$ activates transcription, and doxycycline prohibits this activation.

A luciferase reporter (11), under the regulation of the tet $O$-CMV promoter, was tested for inducible expression when targeted to the Hprt gene. We 


\section{Benchmarks}

used recombinant luciferase, a 61-kDa monomeric protein from fireflies that catalyzes the oxidation of beetle luciferin with the concomitant production of light. Since luciferase is monomeric and does not require posttranslational modification, it serves as a reporter immediately after translation. In addition, luminescence decays rapidly so that accurate time points can be taken. The luciferase assay was performed using the
Promega Luciferase Assay System (Promega, Madison, WI, USA).

The experiment was designed as follows. First, ES cells [Lex1 cells derived from 129SvEvBrd mice (Stratagene, La Jolla, CA, USA)] were deleted for the third exon of Hprt using a vector that replaces exon 3 with a neomycin phosphotransferase selection cassette (neo) flanked by an I-Sce 1 endonuclease site (12) to generate $\mathrm{G} 418^{\mathrm{R}}$ (selection for

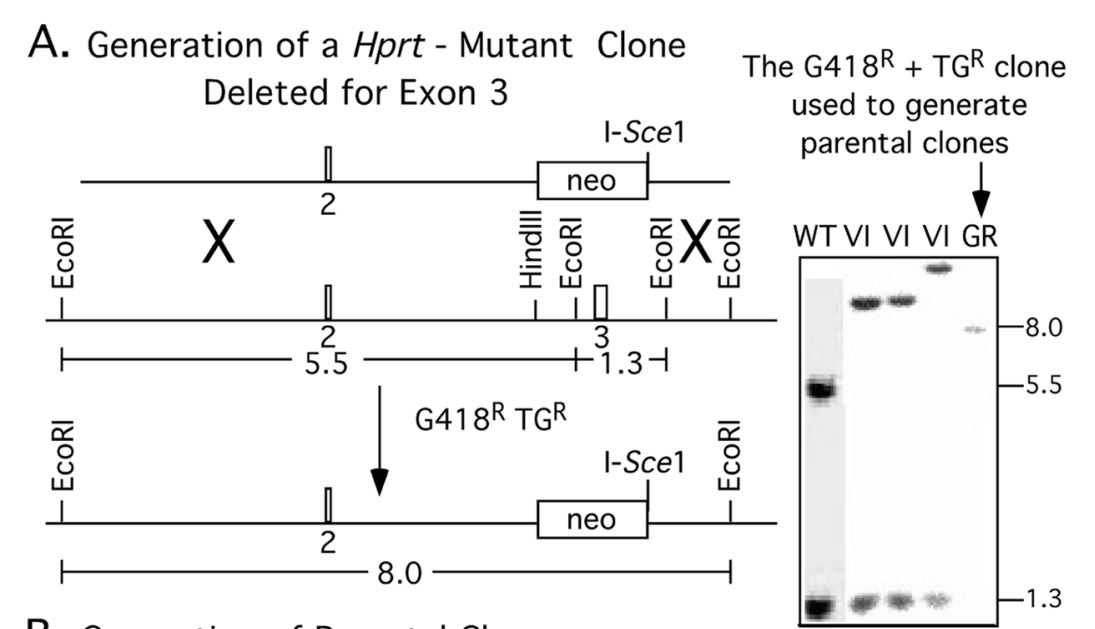

B. Generation of Parental Clones

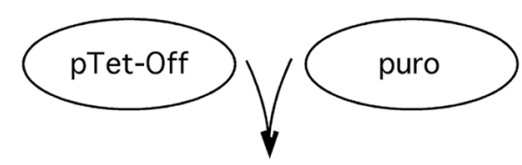

co-transfect pTet-Off and puro into the $\mathrm{G} 418^{\mathrm{R}}+\mathrm{TG}^{\mathrm{R}}$ clone (targeting vector integrated into Hprt by GR) select for puromycin - resistant clones

\section{Generation of Daughter Clones}

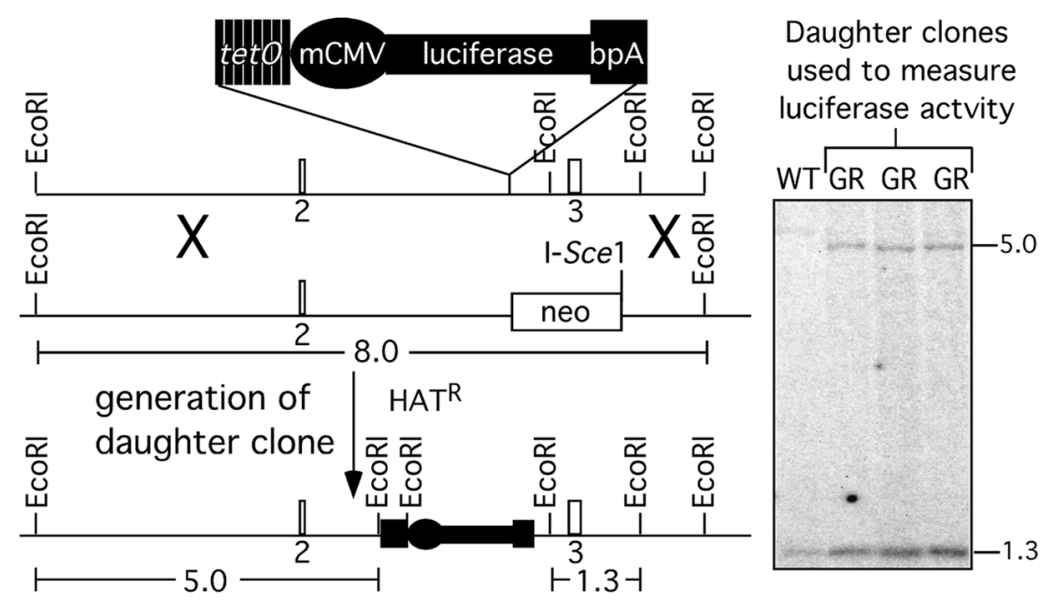

neo expression) $+\mathrm{TG}^{\mathrm{R}}$ clones (Figure 1A). Second, a single $G 418^{R}+T^{R}$ clone that exhibited the correct targeting pattern by Southern analysis was transfected with pTet-Off (BD Biosciences Clontech, Palo Alto, CA, USA), a vector that contains the $t \mathrm{TA}$ expressed by the CMV promoter. In addition, a puromycin $\mathrm{N}$-acetyltransferase selection cassette (puro) was co-transfected with pTet-Off, since pTet-Off contains neo for selection and the $\mathrm{TG}^{\mathrm{R}}$ clone is already resistant to G418. Puromycin-resistant clones were isolat-

Figure 1. Targeted transgenesis strategy. (A) First step: Generation of a clone deleted for Hprt exon 3. Hprt sequences from a HindIII site to an EcoRI site that include exon 3 were replaced with neo (an I-Scel endonuclease site flanks neo). As described previously (14), this targeting vector (10 $\mu \mathrm{g}$ ) was linearized with $\mathrm{BamHI}$ (cuts at the edge of homology) and electroporated into ES cells $(1 \times$ $10^{7}$ cells in $1 \mathrm{~mL}$ PBS at $575 \mathrm{~V} / \mathrm{cm}, 500 \mu \mathrm{F}$ with a Bio-Rad gene pulser II; Bio-Rad Laboratories, Hercules, CA, USA) and selected in G418 (approximately $540 \mu \mathrm{g} / \mathrm{mL}$ active ingredient). After five days in only G418, TG $\left(1 \times 10^{-5} \mathrm{M}\right)$ was added to select for targeted clones. Clones resistant to G418 and TG were expanded and confirmed by Southern analysis (EcoRI-restricted-DNA hybridized to a probe, exons 2 and 3 of $H p r t$ ) for deletion of exon 3 by gene replacement (GR). The wild-type (WT), vector insertion (VI), and GR patterns are shown. Gene replacement is desired and results in a single EcoRI-restricted fragment of 8 $\mathrm{kb}$. Vector insertion is undesired and incorporates the entire vector into Hprt (14). (B) Second step Generation of parental clones. The G418 $\mathrm{R}+\mathrm{TG}^{\mathrm{R}}$ clone that exhibited the GR pattern was expanded and co-electroporated with pTet-Off $(10 \mu \mathrm{g}$ uncut $)$ and puro $(10 \mu \mathrm{g}$ uncut). Puromycin $(3 \mu \mathrm{g} / \mathrm{mL})$ was added $24 \mathrm{~h}$ later, and 20 puromycin-resistant colonies were picked one week later. (C) Third step: Generation of daughter clones. A luciferase reporter was targeted to Hprt by a second gene replacement event and has a tetO-mCMV promoter and a bovine growth hormone polyadenylation site (bpA) (15). The targeting vector (10 $\mu \mathrm{g}$ uncut) and the I-Sce 1 endonuclease expression vector $(30 \mu \mathrm{g}$ uncut) were co-transfected into ES cells (as described in part A) (12). Alternatively, the targeting vector $(10 \mu \mathrm{g}$ cut at the edge of homology with $K p n I)$ was transfected into ES cells. Clones of ES cells that corrected Hprt were selected in HAT ( $1 \mathrm{mM}$ sodium hypoxanthine, $4 \mu \mathrm{M}$ aminopterin $0.16 \mathrm{mM}$ thymidine) media. $\mathrm{HAT}^{\mathrm{R}}$ colonies were picked and expanded while maintaining HAT selection. The I-Sce 1 endonuclease improved the recovery of HAT-resistant clones by about 100 -fold (about 100 compared to about 1 HAT-resistant colony per electroporation). Genomic DNA, isolated from HAT-resistant colonies, was restricted with $E c o$ RI and hybridized to exons 2 and 3 from Hprt cDNA to confirm gene replacement $(8-\mathrm{kb}$ fragment is converted into 5 - and $1.3-\mathrm{kb}$ fragments). All HAT-resistant colonies were correctly targeted by gene replacement. 
Table 1. Mean and Range of Luciferase Activities for Daughter Clones

\begin{tabular}{|ccc|}
\hline Parental Clone No. & Mean Activity & Range of Activity \\
\hline 1 & $6980+/-15854$ & $155-29321$ \\
2 & $47+/-20$ & $16-50$ \\
3 & $690+/-694$ & $47-1322$ \\
5 & $57751+/-28555$ & $6370-101904$ \\
6 & $1002+/-981$ & $142-2474$ \\
7 & $89+/-53$ & $28-167$ \\
8 & $3653+/-2884$ & $451-8778$ \\
9 & $795+/-335$ & $462-1402$ \\
13 & $257+/-227$ & $53-711$ \\
19 & $179+/-91$ & $34-309$ \\
& Luciferase activities were determined for daughter clones generated from 10 \\
parental clones. Three to five daughter clones were observed from each parental \\
clone. The mean counts with standard errors and the range of counts are shown \\
for daughter clones generated from each parental clone. The counts are arbitrary. \\
Luciferase activity was determined by subtracting the counts in the off state (plus \\
doxycycline) from the counts in the on state (minus doxycycline). \\
\hline
\end{tabular}

ed and expanded. These are the parental clones (Figure 1B). Third, for 20 parental clones, a luciferase reporter was targeted to Hprt. This vector contains the luciferase reporter in the second intron of Hprt. Upon recombination, Hprt exon 3 was restored, and these clones were selected in HAT media. Recombination was facilitated using an I-Sce 1 endonuclease expression vector that generated a DNA doublestrand break at the I-Sce 1 endonuclease site adjacent to neo in the Hprt gene (12). The use of the I-Sce 1 endonuclease system increased the generation of HAT $^{\mathrm{R}}$ clones by about 100 -fold (data not shown). These are the daughter clones, and they have normal morphology (Figure 1C).

The mean and range of luciferase activity were determined for $3-5$ of the daughter clones generated from 10 parental clones. Table 1 shows the mean luciferase activity for daughter clones generated from each parental clone. In addition, Table 1 shows the range of luciferase activity for daughter clones generated from each parental clone. For all daughter clones, luciferase activity was observed in the absence but not the presence of doxycycline; thus, the system is inducible and not leaky. However, the variance of inducible luciferase activity was great, suggesting variable levels of $t$ TA ex- pression ( $t \mathrm{TA}$ is in a different location for each parental clone). In addition, the range of luciferase activity was great among the daughter clones derived from a single parental clone, suggesting that either a subpopulation of cells turned off $t \mathrm{TA}$ and/or luciferase or that each daughter clone expressed $t \mathrm{TA}$ and/or luciferase at different levels. Even though the range was great, the variance between individual daughter clones was consistent when compared on different days and was not due to technical variables (not shown).

Inducible transgenesis, as described here, could be optimized by integrating the $t$ TA adjacent to an endogenous promoter by knock-in (13). The knock-in approach would reduce unwanted side effects associated with random integration of transgenes. First, knock-in of the $t$ TA would eliminate the potential unwanted side effect of altering an unknown gene that requires the analysis of multiple clones. Second, knock-in of the $t \mathrm{TA}$ would eliminate the potential unwanted side effect of variable expression levels that may occur because of the formation of heterochromatin or methylation. Alternatively, from another perspective, random integration of pTet-Off may offer an advantage for the investigator interested in a wide range of expression levels.

Targeted transgenesis, by disruption of the Hprt gene (9) or by correction of the Hprt gene (described in Figure 1), is suitable for the expression of transgenes (8). Targeted transgenesis at Hprt enables throughput because of the easy selection strategy to isolate targeted clones. In addition to constitutive expression and promoter-regulated expression, targeted transgenesis at the Hprt gene is suitable for induced expression as shown for the tetO-CMV promoter expressing the luciferase reporter.

\section{REFERENCES}

1.Palmiter, R.D. and R.L. Brinster. 1986 Germ-line transformation of mice. Annu. Rev. Genet. 20:465-499.

2.Woychik, R.P., T.A. Stewart, L.G. Davis, P. D'Eustachio, and P. Leder. 1985. An inherited limb deformity created by insertional mutagenesis in a transgenic mouse. Nature 318:36-40.

3.al-Shawi, R., J. Kinnaird, J. Burke, and J. O. Bishop. 1990. Expression of a foreign gene in a line of transgenic mice is modulated by a chromosomal position effect. Mol. Cell Biol. 10:1192-1198.

4.Reuter, G., M. Giarre, J. Farah, J. Gausz, A. Spierer, and P. Spierer. 1990. Dependence of position-effect variegation in Drosophila on dose of a gene encoding an unusual zinc-finger protein. Nature 344:219-223.

5.Robertson, G., D. Garrick, W. Wu, M. Kearns, D. Martin, and E. Whitelaw. 1995. Position-dependent variegation of globin transgene expression in mice. Proc. Natl. Acad. Sci. USA 92:5371-5375.

6.Bird, A.P. and A.P. Wolffe. 1999. Methylationinduced repression - belts, braces, and chromatin. Cell 99:451-454

7.Sandgren, E.P., R.D. Palmiter, J.L. Heckel, C.C. Daugherty, R.L. Brinster, and J.L. Degen. 1991. Complete hepatic regeneration after somatic deletion of an albumin-plasminogen activator transgene. Cell 66:245-256.

8.Jasin, M., M.E. Moynahan, and C. Richardson. 1996. Targeted transgenesis. Proc. Natl. Acad. Sci. USA 93:8804-8808.

9.Vivian, J.L., W.H. Klein, and P. Hasty. 1999 Temporal, spatial and tissue-specific expression of a myogenin-lacZ transgene targeted to the Hprt locus in mice. BioTechniques 27:154-162.

10.Gossen, M. and H. Bujard. 1992. Tight control of gene expression in mammalian cells by tetracycline-responsive promoters. Proc. Natl. Acad. Sci. USA 89:5547-5551.

11.de Wet, J.R., K.V. Wood, D.R. Helinski, and M. DeLuca. 1986. Cloning firefly luciferase. Methods Enzymol. 133:3-14.

12.Donoho, G. M. Jasin, and P. Berg. 1998. Analysis of gene targeting and intrachromosomal homologous recombination stimulated by genomic double-strand breaks in mouse embryonic stem cells Mol. Cell Biol. 18:4070-4078.

13.Shin, M.K., J.M. Levorse, R.S. Ingram, and S.M. Tilghman. 1999. The temporal requirement for endothelin receptor-B signalling dur- 
ing neural crest development. Nature 402:496-501.

14.Hasty, P., J. Rivera-Perez, C. Chang, and A. Bradley. 1991. Target frequency and integration pattern for insertion and replacement vectors in embryonic stem cells. Mol. Cell Biol. 11:4509-4517.

15.Pfarr, D.S., L.A. Rieser, R.P. Woychik, F.M. Rottman, M. Rosenberg, and M.E. Reff. 1986. Differential effects of polyadenylation regions on gene expression in mammalian cells. DNA 5:115-122.

Address correspondence to Dr. Paul Hasty, Department of Molecular Medicine and Institute of Biotechnology, University of Texas Health Science Center, San Antonio, TX 78245-3207, USA. e-mail: hastye@uthscsa.edu

Received 25 October 2002; accepted 11 December 2002.

\section{Greg Donoho and Paul Hasty Lexicon Genetics The Woodlands, TX, USA}

\section{Direct Comparison of Common Fixation Methods for Preservation of Microtubules in Zebrafish Embryos}

BioTechniques 34:468-472 (March 2003)

Microtubule arrays in zebrafish (Danio rerio) embryos are vital to many developmental processes. Besides their obvious role in mitosis, microtubules are required for epiboly (1-3), furrow formation (4), and the cohesion of postcytokinesis blastomeres (5). As transport lines for regulatory substances and maternal mRNAs, microtubules are also required for axis determination (6) and symmetric and synchronous cleavage (7). The study of microtubule function in both fixed and live embryos is consequently important for understanding the molecular mechanisms underlying numerous developmental processes.

In fixed preparations, immunofluorescence microscopy allows the comparison of microtubule structures in embryos at different stages or comparison of mutant or developmentally modified embryos to wild-type embryos fixed at the same age. Microtubule arrays in thick samples such as embryos are notoriously difficult to preserve. The dynamic nature of the microtubule polymer and sensitivity to calcium make microtubules extremely labile. Furthermore, fixations that work well to preserve microtubules in one cell type or sample may work very poorly in others.

Several fixation methods are routinely used, and there is only anecdotal support for one method over the other. Most of these methods have been adapted from Xenopus protocols, and the anatomy of the zebrafish embryo possesses unique features that may prevent direct extrapolation of these methods. Here we report a comparison of the common fixation methods and make recommendations for the analysis of microtubules in different cell types of the early embryo.

We focus on the visualization of microtubules in three areas of zebrafish embryos: $(i)$ the enveloping layer of cells (EVL), (ii) the deep cells that lie beneath EVL cells, and (iii) the yolk syncytial layer (YSL) (Figure 1). The deep cells of the early embryo will form the tissues of the embryo-proper, while EVL cells are epithelial and form a single-cell covering over the entire embryo. Both the EVL and deep cells sit upon and surround a multinucleated syncytium, the yolk cell. Visualization of these specific domains is valuable in different areas of developmental research.

The embryo develops within a clear sack called the chorion. If the chorion is not removed before fixation, then it must be removed at a later stage for antibody penetration in immunohistochemistry. After fixation, the chorion can be easily removed with forceps. Before fixation, a limited enzymatic pronase digestion can remove the chorion. We tested multiple fixation methods and tested whether removal of the chorion before fixation had any effect.

We examined two common fixation procedures: a simple paraformaldehyde (PFA) fixation and a fixation using microtubule stabilization buffer (MSB). PFA is a widespread fixative used on many different sample types and has been used to prepare zebrafish embryos for immunohistochemistry of many epitopes $(5,8)$, in situ hybridization, and a combination of these two techniques (9). The MSB fixative was originally developed for Xenopus (10) and was later used with slight modifications for zebrafish embryos $(1,11)$. We examined these two fixatives both with and without intact chorions during fixation. We also tested embryos that were permeabilized with Triton ${ }^{\circledR}$ X-100 following de-

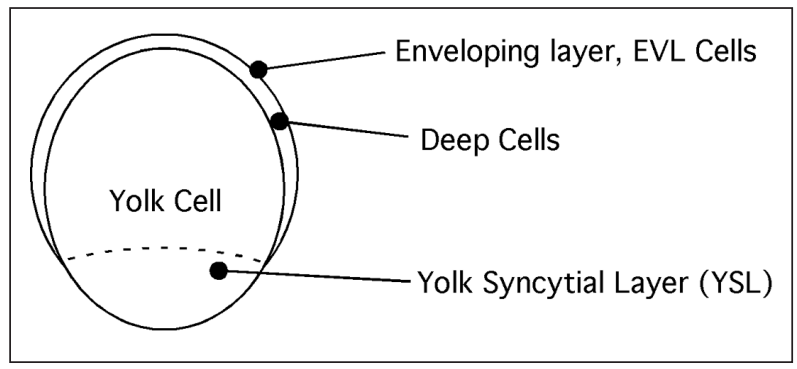

Figure 1. Representation of an 8-h zebrafish embryo, showing the location of the different cell types examined in this study. 